\title{
COVID-Associated Pulmonary Aspergillosis and Its Related Outcomes: A Single-Center Prospective Observational Study
}

\author{
Ahtesham Iqbal ${ }^{1}$, Moazma Ramzan ${ }^{1}$, Aftab Akhtar ${ }^{2}$, Anam Ahtesham ${ }^{3}$, Seemal Aslam ${ }^{4}$, Javeria Khalid ${ }^{5}$ \\ 1. Critical Care, Shifa International Hospital, Islamabad, PAK 2. Pulmonary and Critical Care, Shifa International \\ Hospital, Islamabad, PAK 3. Pharmacy, Bahawal Victoria Hospital, Bahawalpur, PAK 4. Internal Medicine, Quaid-e- \\ Azam Medical College, Bahawalpur, PAK 5. Pharmacy, Shifa International Hospital, Islamabad, PAK
}

Corresponding author: Ahtesham Iqbal, dr.ahtesham@hotmail.co.uk

\section{Abstract}

\section{Background and objective}

Invasive pulmonary aspergillosis (IPA) is a frequent complication among neutropenic patients. It is increasingly being reported in critical coronavirus disease 2019 (COVID-19) patients requiring ICU admission and invasive mechanical ventilation (IMV) and is known as COVID-associated pulmonary aspergillosis (CAPA). We conducted this large prospective observational study to determine the frequency of CAPA and its outcomes in the ICU population.

\section{Methodology}

This was a prospective observational study. We recruited 307 reverse transcription-polymerase chain reaction (RT-PCR)-confirmed cases of severe COVID-19 pneumonia requiring IMV. We excluded those who did not require IMV or had been transferred out to other hospitals. The Chi-square test was applied to find the association between categorical variables. A p-value of $<0.05$ was considered statistically significant.

\section{Results}

Out of the 307 cases of mechanically ventilated COVID-19 pneumonia, 61 had probable CAPA. The median age was 60 years. Malignancy and cirrhosis were significant risk factors associated with CAPA $(p=<0.001$, 0.001 , respectively). Aspergillus fumigatus was detected in $78.7 \%$ of the cases. The median length of ICU stay was 11 days [interquartile range (IQR): 4-14]. Among CAPA cases, 70.5\% developed septic shock and required ionotropic support. Among 61 probable cases of CAPA, 91.8\% did not survive and there was a strong correlation between CAPA and ICU mortality $(\mathrm{p}=0.001)$.

\section{Conclusion}

We concluded that CAPA is a fatal complication of severe COVID-19 pneumonia and is associated with

Review began 07/12/2021 Review ended 07/26/2021 Published 08/07/2021

\section{๑) Copyright 2021}

Iqbal et al. This is an open access article distributed under the terms of the Creative Commons Attribution License CC-BY 4.0., which permits unrestricted use, distribution, and reproduction in any medium, provided the original author and source are credited.
Categories: Internal Medicine, Infectious Disease, Pulmonology

Keywords: covid associated pulmonary aspergillosis, invasive mechanical ventilation, severe covid pneumonia, outcome, probable capa

\section{Introduction}

Invasive pulmonary aspergillosis (IPA) is a fatal disease affecting immunosuppressed individuals with hematological malignancies and recipients of allogeneic bone marrow transplantation who develop acute graft versus host disease [1]. With the development of new treatment modalities and the emergence of novel diseases like influenza and coronavirus disease 2019 (COVID-19), the spectrum of risk factors of IPA has enormously expanded. In a study by Schauwvlieghe et al., influenza was found to be an independent risk factor for IPA, especially in patients who require ICU care [2]. Similarly, COVID-19 is also an independent risk factor for IPA and this entity is known as COVID-associated pulmonary aspergillosis (CAPA) [3]. Many risk factors have been identified to be involved in the pathogenesis of CAPA, which include the use of systemic corticosteroids in acute respiratory distress syndrome (ARDS), severe lung damage, comorbidities, and extensive use of broad-spectrum antibiotics [4]. Like influenza, COVID-19 also disrupts the respiratory epithelium and impairs ciliary dysfunction and the immune regulatory system, thereby facilitating the growth of Aspergillus spp. into tissues [5]. However, the pathogenesis of CAPA is still unknown. It is postulated that intense inflammatory response in COVID-19 results in a decline in lymphocyte count and impairs their function, which leads to increased susceptibility to CAPA in severe COVID-19 pneumonia [4]. The mortality rate associated with CAPA ranges from $66.1 \%$ to $80.6 \%$ [6]. The largest single-center prospective observational study conducted in the ICU population has reported $100 \%$ mortality in CAPA as compared to those without CAPA (44\%) [7]. 
With the proven role of steroids in the treatment of severe COVID-19 pneumonia, fungal and bacterial coinfections are increasingly being reported in the ICU population and causing a huge impact on the outcomes of severe COVID-19 pneumonia. A meta-analysis of 24 studies comprising more than 3,000 severe COVID-19 pneumonia cases has reported $14.3 \%$ bacterial coinfections in ICU [8]. Similarly, CAPA is also increasingly being reported in the ICU population. Recently, 33\% and 26\% cases have been reported in France and Germany respectively $[3,9,10]$. A systematic review has reported a $35 \%$ to $3.8 \%$ incidence of CAPA in severe COVID-19 pneumonia, which included subjects mostly from European countries [11]. A Greek tertiary care referral hospital has reported an incidence of $3.3 \%$ in 179 polymerase chain reaction (PCR)-confirmed cases of COVID-19 pneumonia and, interestingly, none of them had immunosuppression [12]. Another study has reported a $27.7 \%$ incidence of probable cases after four days of invasive mechanical ventilation (IMV) [13].

Epidemiological studies conducted to evaluate the incidence of CAPA may not represent the true incidence of infection as most of them are small-sized and results are inconsistent in different parts of the world. Even though a retrospective study conducted at the national level reported $21.7 \%$ CAPA cases in 147 cases of COVID-19 pneumonia [14], the true incidence and mortality rates of CAPA remain underestimated in the ICU population since its results cannot be generalized to the larger population due to its small sample size. An expert panel has recently suggested conducting more epidemiological studies to evaluate the incidence of CAPA in the ICU population. In light of this, we conducted this prospective observational study to evaluate the frequency of CAPA and its associated outcomes in the ICU.

\section{Materials And Methods}

This was a prospective observational study conducted at the Medical ICU of the Shifa International Hospital, Islamabad, which is a large tertiary care center in Pakistan. The study period spanned from June 2020 to May 2021. Ethical approval was obtained from the Institutional Review and Ethical Committee of the Shifa International Hospital, Islamabad (IRB \#: 158-978-2020). Written consent was taken from surrogates of study participants. After fulfilling inclusion and exclusion criteria, 307 cases were enrolled. The inclusion criteria were as follows: adult patients of $\geqslant 18$ years, with RT-PCR-confirmed cases of COVID-19 pneumonia, admitted to the ICU with hypoxemic respiratory failure requiring IMV. We excluded those patients who did not require IMV, those who were RT-PCR-negative for COVID-19, those who did not consent to participate, and those who had transferred out to other hospitals. We followed up on our patients till the time of discharge from ICU or death.

Based on worsening respiratory symptoms with or without fever, we obtained chest X-rays and galactomannan for all patients along with other necessary investigations. Serum galactomannan testing was performed using Platelia ${ }^{\mathrm{TM}}$ Aspergillus kit (Bio-Rad Laboratories, Hercules, CA) with a cut-off value of $>0.5$. The tracheal aspirate was cultured for fungus. Probable CAPA was diagnosed based on worsening respiratory symptoms, radiological infiltrates, serum galactomannan $>0.5$, and fungal growth on tracheal aspirate. This definition was consistent with consensus criteria for research and clinical guidance for defining CAPA and proposed by the European Confederation of Medical Mycology/International Society for Human and Animal Mycology (ECMM/ISHAM) [15]. In addition to steroids, all patients received the usual standard of care including antibiotics. The infectious disease team was taken on board to decide about the inclusion of remdesivir, tocilizumab, or both. The decision for IMV was based on clinical condition and resuscitation code status. The mortality predictor scores like Acute Physiology and Chronic Health Evaluation (APACHE II), Sequential Organ Failure Assessment (SOFA), and Nutrition Risk in Critically ill (NUTRIC) scores were calculated at the time of admission to the ICU. All patients diagnosed with CAPA were treated with either oral voriconazole or amphotericin. Data including demographics and clinical, radiologic, and treatment outcomes were collected on a preformed structured questionnaire.

The primary measure was to evaluate the frequency of CAPA. Our secondary outcome was to evaluate mortality in CAPA. Mortality was defined as death during the stay in the ICU. Survival was defined as discharge from the ICU.

\section{Statistical analysis}

Results were analyzed using SPSS Statistics version 20 (IBM, Armonk, NY). The Shapiro-Wilk test was used to check the normality of continuous variables. Age, having normal distribution, was presented as mean \pm standard deviation and other variables were presented as median [interquartile range (IQR)], being nonnormal in distribution. Categorical variables like gender, comorbidities, diabetes mellitus, and outcomes were presented in percentages. A Chi-square test was applied to find the association between CAPA and ICU mortality. A p-value of $<0.05$ was considered statistically significant.

\section{Results}

We prospectively analyzed 307 patients in total. The flow diagram about the study participants is presented in Figure 1. 


\section{Cureus}

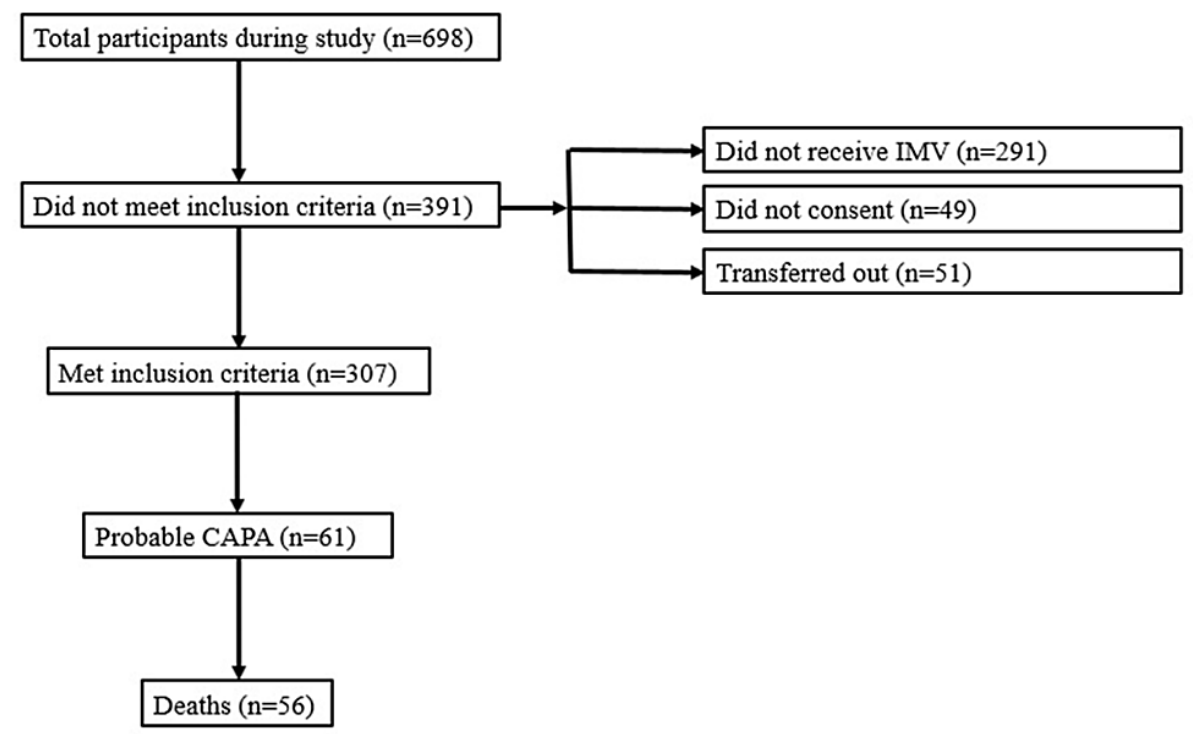

\section{FIGURE 1: Flow diagram about the study participants}

CAPA: COVID-19-associated pulmonary aspergillosis; IMV: invasive mechanical ventilation

The median age of our study participants was 60 years. Out of 307 participants, $19.9 \%(n=61)$ were diagnosed as probable cases of CAPA. Characteristics of probable CAPA cases are shown in Table 1. Out of these 61, $47.5 \%$ were females and $52.5 \%$ were males. Malignancy and cirrhosis of the liver were identified as statistically significant risk factors associated with CAPA $(p=<0.001,0.001$, respectively), whereas diabetes mellitus and multiple comorbidities did not have a statistically significant association ( $\mathrm{p}=0.55,0.54$, respectively). All probable cases of CAPA had received dexamethasone $6 \mathrm{mg} / \mathrm{kg} / \mathrm{day}$ or methylprednisolone $1-2 \mathrm{mg} / \mathrm{kg} /$ day and also received voriconazole as a curative treatment. Mean values of clinical severity scores and characteristics of CAPA patients are given in Table 1. The culture of tracheal aspirates showed that Aspergillus fumigatus was the most common species to cause CAPA followed by Aspergillus flavus (78.7\% vs. 16.4\%); $57.4 \%$ of total probable CAPA cases received remdesivir and $54.1 \%$ were given tocilizumab. The median length of ICU stay was recorded as 11 days (IQR: 4-14). Among CAPA cases, $70.5 \%$ developed septic shock and required ionotropic support. We observed $60.9 \%(\mathrm{n}=187)$ mortality among 307 study participants. Our results showed that $91.8 \%(\mathrm{n}=56)$ did not survive in the cohort of probable CAPA, and there was a strong association between CAPA and mortality $(\mathrm{p}=0.001)$. 


\section{Cureus}

Variables

Values $(n=61)$

Demographic data

Age in years (mean $\pm S D$ )

$60.7 \pm 8.7$

Gender ratio (male:female)

29:32

Smoking history $(n=80), \%(n)$

$27.9 \%(n=17)$

Hypertension, \% (n)

$49.2 \%(n=30)$

Risk factors for CAPA, \% (n)

DM

$73.8 \%(n=45)$

Cirrhosis

$8.2 \%(n=5)$

$\geq 2$ comorbidities

$90.2 \%(n=55)$

Malignancy

$21.3 \%(n=13)$

Clinical variables

APACHE II, median (IQR)

$14(10-21)$

NUTRIC score, median (IQR)

SOFA score, median (IQR)

$4(4-7)$

SAPS, median (IQR)

$33(27-45)$

Aspergillus fumigatus, \% (n)

$78.7 \%(n=48)$

Aspergillus flavus, \% (n)

$16.4 \%(n=10)$

Aspergillus terreus, \% (n)

$3.3 \%(n=2)$

Aspergillus niger, \% (n)

$1.6 \%(n=1)$

Treatment and clinical course

Remdesivir, \% (n)

$57.4 \%(n=35)$

Tocilizumab, \% (n)

$54.1 \%(n=33)$

Dexamethasone/methylprednisolone, \% (n)

$100 \%(n=61)$

Voriconazole, \% (n)

$100 \%(n=61)$

Lipid formulation amphotericin, \% (n)

$3.3 \%(n=2)$

Vasopressors, \% (n)

$70.5 \%(n=43)$

ICU days, median (IQR)

$11(4-14)$

Death, \% (n)

$91.8 \%(n=56)$

\section{TABLE 1: Characteristics of probable CAPA cases}

CAPA: COVID-19-associated pulmonary aspergillosis; DM: diabetes mellitus; APACHE: Acute Physiology and Chronic Health Evaluation; NUTRIC: Nutrition Risk in Critically III; SOFA: Sequential Organ Failure Assessment; SAPS: Simplified Acute Physiology Score; ICU: intensive care unit; SD: standard deviation

\section{Discussion}

This was a prospective observational study that aimed to estimate the frequency of CAPA and associated mortality in our ICU population. Mean values of APACHE II, SOFA, NUTRIC, and SAPS II indicated the severity of disease in our study participants. We detected $19.9 \%$ cases of probable CAPA. The incidence of CAPA in our population was greater than the $10.1 \%$ rate reported in a retrospective study conducted in the USA. Many other studies from Europe have reported an incidence of $26.0 \%$ to $33 \%[3,9,10,13]$. All these studies had a small sample size, and hence their results cannot be generalized to a larger population. Such variations in the incidence of CAPA are bound to happen due to variable infection control practices, ICU 
designs, and risk factors in the study populations. Aspergillus spp. is a common colonizer of the airway and can progress to overt infection in the ICU population because the use of antibiotics and steroid exposure can alter the host immune response [16-18]. IPA, an entity representing Aspergillus infection in non-COVID-19 patients, occurs most commonly in those who undergo chemotherapy for hematological malignancies. Our analysis showed that malignancy and cirrhosis were significant risk factors associated with CAPA infection. The association of malignancy and cirrhosis with the development of CAPA has not been adequately studied; CAPA is a relatively new entity and a lot of research is ongoing to explore its associated risk factors. A systematic review of 22 studies comprising only 85 patients has described the risk factors of CAPA and identified only two patients with malignancy [11]. It explains the scarcity of data and supports the work of Koehler et al., who has explored COVID-19 as an independent risk factor of CAPA [3]. Another important risk factor for the development of CAPA is tocilizumab, which is an interleukin 6 (IL-6) inhibitor and is known to cause multiple co-bacterial and co-fungal infections in the ICU population [19]. In our cohort of CAPA cases, $54.1 \%$ had received tocilizumab, and all had received corticosteroids, which are known to cause immunosuppression and paves the way for the growth of Aspergillus and tissue invasion to cause CAPA [2023].

We observed 91.8\% mortality in probable CAPA cases. It was beyond our study objective to explore factors associated with increased mortality in our cohort of probable CAPA cases. A meta-analysis of 28 studies has shown mortality of only $54.9 \%$ in the ICU population. This meta-analysis included 18 retrospective studies, and case definitions of CAPA were based on European Organization for Research and Treatment of Cancer/Mycosis Study Group (EORTC/MSG) criteria, which could have underestimated the incidence of CAPA as most ICU patients with CAPA lack traditional risk factors to meet the criteria of CAPA. In contrast, we diagnosed all cases of CAPA by satisfying the recent criteria proposed by Koehler et al. Another study from Italy has compared influenza-associated pulmonary aspergillosis with CAPA and has reported a mortality rate of $40-60 \%$ [24]. It is a subject of debate whether this mortality is attributed to CAPA itself or complicated COVID-19 pneumonia. A literature review was also inconclusive in this regard. A prospective study of 108 cases, after adjusting for confounding factors, has reported an increased association of mortality with CAPA (OR: 3.53) and a decrease in mortality with the use of antifungals [13]. In contrast, a large prospective US study did not find any association between increased mortality and CAPA despite poor outcomes in terms of length of stay and duration of IMV. Similarly, another case series of putative CAPA has shown decreased mortality even with the absence of antifungals. More robust evidence is needed to ascertain the impact of CAPA on the outcomes in the ICU population.

This study has some limitations. Firstly, it was a single-center study. Secondly, we did not study the contribution of CAPA to mortality in severe COVID-19 pneumonia as it was beyond our objective of the study. Finally, the galactomannan test in the bronchoalveolar fluid was not available at our study center, and hence we measured galactomannan in serum. Studies have suggested that the sensitivity of galactomannan is $40 \%$ as compared to that of the bronchoalveolar fluid, which is $90 \%$ [25]. This could have led to a potential bias of underreporting relating to the actual number of probable cases of CAPA in our study population. Larger comparative studies are required to calculate the impact of CAPA on mortality in ventilated patients. However, the large sample size of our study and its prospective design are the two major strengths of our study. Given our large sample size, our results can be generalized to critical COVID-19 patients requiring IMV.

\section{Conclusions}

Based on our findings, CAPA is a frequent complication of severe COVID-19 pneumonia in the ICU population and is associated with an increased mortality rate. Prompt recognition and treatment may lead to favorable outcomes. We recommend further research to investigate its impact on ICU mortality in critical COVID-19 patients.

\section{Additional Information \\ Disclosures}

Human subjects: Consent was obtained or waived by all participants in this study. Institutional Review Board and Ethics Committee, Shifa International Hospital, Islamabad issued approval 137-957-2020. This study has been approved by the Institutional Review Board and Ethics Committee, Shifa International Hospital, Islamabad. Animal subjects: All authors have confirmed that this study did not involve animal subjects or tissue. Conflicts of interest: In compliance with the ICMJE uniform disclosure form, all authors declare the following: Payment/services info: All authors have declared that no financial support was received from any organization for the submitted work. Financial relationships: All authors have declared that they have no financial relationships at present or within the previous three years with any organizations that might have an interest in the submitted work. Other relationships: All authors have declared that there are no other relationships or activities that could appear to have influenced the submitted work.

\section{Acknowledgements}

We would like to acknowledge Dr. Muhammad Irfan from Agha Khan Hospital and Dr. Ayyaz Mir from Shifa 


\section{References}

1. Verweij PE, Rijnders BJ, Brüggemann RJ, et al.: Review of influenza-associated pulmonary aspergillosis in ICU patients and proposal for a case definition: an expert opinion. Intensive Care Med. 2020, 46:1524-35. 10.1007/s00134-020-06091-6

2. Schauwvlieghe AF, Rijnders BJ, Philips N, et al.: Invasive aspergillosis in patients admitted to the intensive care unit with severe influenza: a retrospective cohort study. Lancet Respir Med. 2018, 6:782-92. 10.1016/S2213-2600(18)30274-1

3. Koehler P, Cornely OA, Böttiger BW, et al.: COVID-19 associated pulmonary aspergillosis. Mycoses. 2020, 63:528-34. 10.1111/myc.13096

4. Arastehfar A, Carvalho A, van de Veerdonk FL, et al.: COVID-19 associated pulmonary aspergillosis (CAPA) -from immunology to treatment. J Fungi (Basel). 2020, 6:91. 10.3390/jof6020091

5. Thompson Iii GR, Cornely OA, Pappas PG, et al.: Invasive aspergillosis as an under-recognized superinfection in COVID-19. Open Forum Infect Dis. 2020, 7 :ofaa242. 10.1093/ofid/ofaa242

6. Cui N, Wang H, Long Y, Liu D: $\mathrm{CD}^{+} \mathrm{T}$-cell counts: an early predictor of risk and mortality in critically ill immunocompromised patients with invasive pulmonary aspergillosis. Crit Care. 2013, 17:R157. 10.1186/cc12836

7. Machado M, Valerio M, Álvarez-Uría A, et al.: Invasive pulmonary aspergillosis in the COVID-19 era: an expected new entity. Mycoses. 2021, 64:132-43. 10.1111/myc.13213

8. Langford BJ, So M, Raybardhan S, et al.: Bacterial co-infection and secondary infection in patients with COVID-19: a living rapid review and meta-analysis. Clin Microbiol Infect. 2020, 26:1622-9. 10.1016/j.cmi.2020.07.016

9. Alanio A, Dellière S, Fodil S, Bretagne S, Mégarbane B: Prevalence of putative invasive pulmonary aspergillosis in critically ill patients with COVID-19. Lancet Respir Med. 2020, 8:e48-9. 10.1016/S22132600(20)30237-X

10. van Arkel AL, Rijpstra TA, Belderbos HN, van Wijngaarden P, Verweij PE, Bentvelsen RG: COVID-19associated pulmonary aspergillosis. Am J Respir Crit Care Med. 2020, 202:132-5. 10.1164/rccm.2020041038LE

11. Apostolopoulou A, Esquer Garrigos Z, Vijayvargiya P, Lerner AH, Farmakiotis D: Invasive pulmonary aspergillosis in patients with SARS-CoV-2 infection: a systematic review of the literature. Diagnostics (Basel). 2020, 10:807. 10.3390/diagnostics10100807

12. Paramythiotou E, Dimopoulos G, Koliakos N, Siopi M, Vourli S, Pournaras S, Meletiadis J: Epidemiology and incidence of COVID-19-associated pulmonary aspergillosis (CAPA) in a Greek tertiary care academic reference hospital. Infect Dis Ther. 2021, 10:1779-92. 10.1007/s40121-021-00486-8

13. Bartoletti M, Pascale R, Cricca M, et al.: Epidemiology of invasive pulmonary aspergillosis among COVID-19 intubated patients: a prospective study (Epub ahead of print). Clin Infect Dis. 2020, 10.1093/cid/ciaa1065

14. Nasir N, Farooqi J, Mahmood SF, Jabeen K: COVID-19-associated pulmonary aspergillosis (CAPA) in patients admitted with severe COVID-19 pneumonia: an observational study from Pakistan. Mycoses. 2020, 63:766-70. 10.1111/myc.13135

15. Koehler P, Bassetti M, Chakrabarti A, et al.: Defining and managing COVID-19-associated pulmonary aspergillosis: the $2020 \mathrm{ECMM} / \mathrm{ISHAM}$ consensus criteria for research and clinical guidance. Lancet Infect Dis. 2021, 21:e149-62. 10.1016/S1473-3099(20)30847-1

16. Lionakis MS, Kontoyiannis DP: Glucocorticoids and invasive fungal infections. Lancet. 2003, 362:1828-38. 10.1016/S0140-6736(03)14904-5

17. Meersseman W, Lagrou K, Maertens J, Van Wijngaerden E: Invasive aspergillosis in the intensive care unit. Clin Infect Dis. 2007, 45:205-16. 10.1086/518852

18. van de Veerdonk FL, Kolwijck E, Lestrade PP, et al.: Influenza-associated aspergillosis in critically ill patients. Am J Respir Crit Care Med. 2017, 196:524-7. 10.1164/rccm.201612-2540LE

19. Deana C, Vetrugno L, Bassi F, De Monte A: Tocilizumab administration in COVID-19 patients: water on the fire or gasoline?. Med Mycol Case Rep. 2021, 31:32-4. 10.1016/j.mmcr.2021.01.002

20. Cunha C, Gonçalves SM, Duarte-Oliveira C, et al.: IL-10 overexpression predisposes to invasive aspergillosis by suppressing antifungal immunity. J Allergy Clin Immunol. 2017, 140:867-70. 10.1016/j.jaci.2017.02.034

21. Rutsaert L, Steinfort N, Van Hunsel T, et al.: COVID-19-associated invasive pulmonary aspergillosis. Ann Intensive Care. 2020, 10:71. 10.1186/s13613-020-00686-4

22. McCullers JA: The co-pathogenesis of influenza viruses with bacteria in the lung . Nat Rev Microbiol. 2014, 12:252-62. 10.1038/nrmicro3231

23. Deana C: Dexamethasone/tocilizumab: herpes virus reactivation and COVID-19-associated pulmonary Aspergillosis secondary to off label use: case report. Reactions Weekly. 2021, 1852:165. 10.1007/s40278021-94618-0

24. Lamoth F, Lewis RE, Walsh TJ, Kontoyiannis DP: Navigating the uncertainties of COVID-19 associated aspergillosis (CAPA): a comparison with influenza associated aspergillosis (IAPA) (Epub ahead of print). J Infect Dis. 2021, 10.1093/infdis/jiab163

25. Wu Z, Wang L, Tan L, Wu J, Chen Z, Hu M: Diagnostic value of galactomannan in serum and bronchoalveolar lavage fluid for invasive pulmonary aspergillosis in non-neutropenic patients. Diagn Microbiol Infect Dis. 2021, 99:115274. 10.1016/j.diagmicrobio.2020.115274 